\title{
Das Mammut von Taimyr
}

(Neue Erkenntnisse zur Ókologie des sibirischen Mammuts)

Von N. PolutofF, Tübingen

Mit $2 \mathrm{Abb}$. im Text

Im Herbst 1948 entdeckten zwei Mitglieder einer Polarstation Mammutreste auf der Halbinsel Taimyr in Nordsibirien. Etwas später traf ein Stoßzahn dieses Mammuts im Paläontologischen Institut der Akademie der Wissenschaften ein. Das Mammut wurde an einem auf den geographischen Karten bisher unbekannten Fluß gefunden, dem der Namen Mamontovaja-Fluß (=Mammut-Fluß) gegeben wurde (vgl. Abb. 1). Diese Entdeckung ist wohl keine große Überraschung, da das Mammut in Nordsibirien bekanntlich sehr stark, wie in keinem anderen Teil der Welt, verbreitet war. Trotzdem ist jeder neue Fund von großem Interesse für die Erweiterung unserer Kenntnisse über die Lebensweise dieser ausgestorbenen Riesen.

Den ersten direkten Hinweis (1704) auf den Fund einer Mammutleiche in Sibirien, nämlich am Fluß Jenissei bei Turuchansk, verdanken wir IsBRAND IDES, der als Gesandter Peters des Großen eine Reise durch Sibirien nach China unternahm. Auf Taimyr selbst wurden Mammutreste bereits 1843 von Middendorf am Fluß Taimyra, annähernd auf $75^{\circ}$ n. Br., und später (1889) ein ganzes Mammut am Fluß Kl. Balachna, nahe der Chatanga-Bucht, ungefähr $73^{\circ}$ n. Br., gefunden.

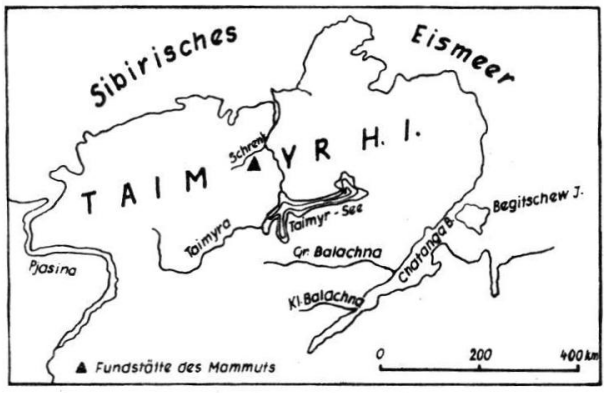

Abb. 1. Die Fundstätte des Taimyr-Mammuts

Die Geschichte der Ausgrabung des 1948 gefundenen Mammuts ist kurz folgende: Im Januar 1949 wurde bei der russischen Akademie der Wissenschaften unter dem Vorsitz des Akademiemitgliedes Prof. E. N. PAvlovskij eine Sonderkommission zur Ausgrabung des Taimyr-Mammuts ins Leben gerufen. Die Kommission sandte zunächst einen Erkundungstrupp voraus, dem dann eine vollständige Expedition folgen sollte ${ }^{1}$ ). Der Erkundungstrupp bestand aus Prof. L. A. Pontenko (Zoologe und Leiter des Trupps), Prof. B. A. Tiсномrnoff (Botaniker), A. I. Popov (Geologe) sowie vier wissenschaftlichen und technischen Assistenten und Arbeitern, im ganzen 15 Mann.

Ende Mai 1949 verließ der Erkundungstrupp Moskau und begab sich auf dem Luftwege zunächst nach Archangelsk. Von dort ging der Weg entlang der Eismeerküste weiter. Zwei Tage nach dem Verlassen Moskaus landete der Trupp in der Bucht Wostotschnaja auf Taimyr. Ausgerïstet mit allem Nötigen für das Leben in der Arktis, begab sich der Trupp am 10. 6. auf Lastwagen mit Raupenantrieb ins Innere der

1) Von der Entsendung der vollständigen Expedition wurde später abgeschen, da der Erkundungstrupp allein die Mammutreste bergen konnte. 
Taimyr-Halbinsel. Nach Zurücklegung von etwa 180 bis $200 \mathrm{~km}$ erreichte die Expedition am 13. 6. den Fluß Mamontovaja, einen Zufluß des Flusses Schrenk. Erst nach großen Anstrengungen gelang es den Forschern, die Fundstätte des Mammuts zu finden, da das 1948 hier hinterlassene Erkennungszeichen inzwischen durch Stürme und Schneeverwehungen vernichtet wurde. Am 15. Juni wurden die Ausgrabungen der Mammutleiche im vollen Umfange in Angriff genommen. Der erhärtete, dichte Schnee ließ sich nur mit großer Mühe wegräumen. Mit der Tiefe wurden die Ausgrabungen noch schwieriger, da man hier auf den ewigen Frostboden stieß. Jetzt war besondere Aufmìerksamkeit geboten, denn bei einem unvorsichtigen Schlag mit der Hacke konnten die Skeletteile des Mammuts beschädigt werden.

Zunächst wurde der Schädel mit einem Stoßzahn herausgenommen und zum Trocknen an das Ufer des Flusses gelegt (der zweite Zahn befand sich, wie erwähnt, schon im Museum). Doch mußten weitere Ausgrabungen bald eingestellt werden, da die Zeit des Hochwassers heranrückte, welches das Skelettgerïst beschädigen und fortschwemmen konnte. Deshalb wurde die Grube mit Flußgeröll aufgefüllt. Am 10. Juli stieg das Wasser im Fluß tatsächlich so stark, daß es die Grube ganz überdeckte. Die Ausgrabungen konnten erst am 5. August wiederaufgenommen werden. Die Arbeiten gestalteten sich jetzt noch schwieriger, da man bei der Vertiefung der Grube dauernd mit ständig einsickerndem Wasser aus dem Fluß Mamontovaja kämpfen mußte. Doch gelang es den Forschern Mitte August, alle Skeletteile ans Ufer zu ziehen. Sie wurden sorgfältig verpackt, zunächst mit Flugzeugen an die Küste des Eismeeres und dann ebenfalls auf dem Luftwege nach Leningrad gebracht, wo sie mit den an der Fundstätte gesammelten Bodenproben und Pflanzenresten Mitte Oktober 1949 eingetroffen waren.

Das eingehende Studium der Einbettungsverhältnisse der Mammutreste und einige Begleiterscheinungen führten die Forscher zur einstimmigen Erkenntnis, daß sich das ausgegrabene Skelett auf sekundärer L a gerstätte befand. Ursprünglich war die Mammutleiche auf der Steilwand der ersten (oder zweiten?) Aueterrasse des Flusses Mamontovaja, in unmittelbarer Nähe seiner jetzigen Lagerstätte, eingebettet. Im Laufe der Zeit wurde diese Steilwand vom Fluß unterhöhlt und stürzte zusammen mit der Mammutleiche auf das heutige Flußufer herunter. Der gute Erhaltungszustand der Knochen und die Anwesenheit einiger Weichteile dürften dafür sprechen, daß diese Umbettung der Leiche vor geologisch relativ kurzer Zeit stattgefunden hat.

Welche Tatsachen sprechen nun eigentlich für eine spätere Umbettung des Mammuts? Auf den Knochen und den leider nur wenigen erhalten gebliebenen Weichteilen des Tieres sind zahlreiche Bruchstücke eines anhaftenden sandig-tonigen Torfgesteins, insgesamt etwa 30 bis $35 \mathrm{~kg}$, gefunden worden. Dieses Gestein besteht aus einer Wechsellagerung von feinsandigen und schlammigen Lagen mit solchen, in denen Pflanzenreste reichlich angesammelt sind. Die Dicke einzelner Lagen schwankt, zwischen einigen Millimetern und $2 \mathrm{~cm}$. Das genannte Gestein, welches den Geröllmassen, aus denen das Skelett geborgen wurde, völlig fremd ist, weist große Ähnlichkeit mit den Torfablagerungen der 1. Aueterrasse des Flusses Mamontovaja nahe der sekundären Lagerstätte des Mamimuts auf. Auch die oben erwähnten Pflanzenreste sprechen zu Gunsten dieser Auffassung, da sie mit denen der Aueterrasse identisch sind.

Die gesammelten Beobachtungen lassen als sicher annehmen, daß das TaimyrMammut zur Zeit der Bildung der ersten Aueterrasse gelebt hat. Die primäre Einbettung des Mammutkadavers in Torfablagerungen der ersten Aueterrasse, der Erhaltungszustand seiner Weichteile, die Bleichung der Haare, wie sie für Tierkadaver charakteristisch ist, die lange Zeit im Wasser gelegen haben, sprechen dafür, daß das TaimyrMammut in einem Sumpfbecken seinen Tod fand, vielleicht nach einem Kampf mit einem stärkeren Gegner, worauf Beschädigungen an der rechten Schulter und am Schulterblatt hinweisen. 


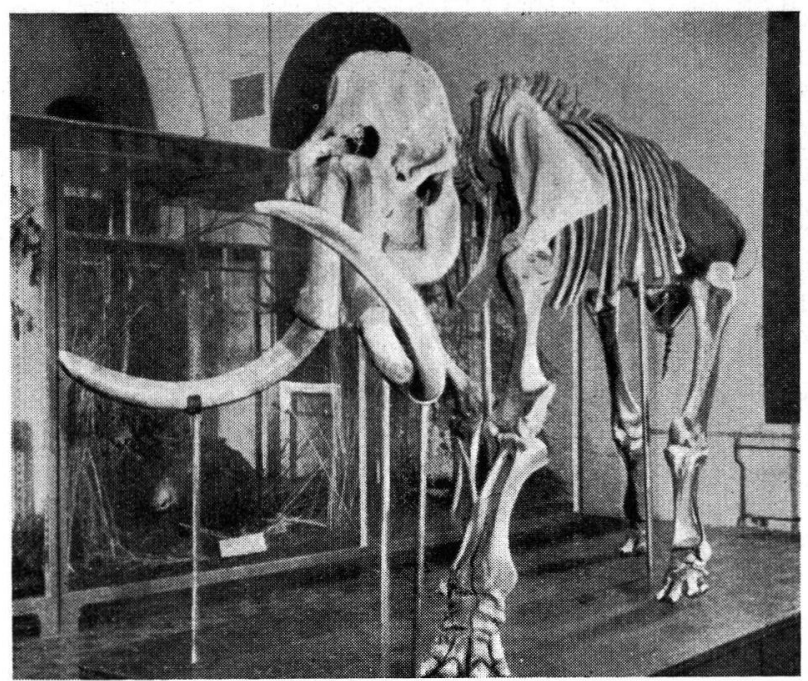

Abb. 2. Das Skelett des Taimyr-Mammuts im Zoologischen Institut der Akad. der Wiss.

Das Skelett des Taimyr-Mammuts erwies sich hinsichtlich der Vollzähligkeit und des Erhaltungszustandes der Knochen wohl als das beste unter den in russischen $\mathrm{Mu}$ seen vorhandenen Exemplaren. Das Taimyr-Mammut ist $310 \mathrm{~cm}$ lang, $250 \mathrm{~cm}$ hoch (vom Boden bis zum Scheitel). Ausgezeichnet sind die Stoßzähne erhalten. Der rechte, 1948 ins Museum eingelieferte Stoßzahn war schon zu Lebzeiten des Tieres beschädigt. Er ist $185 \mathrm{~cm}$ lang und wiegt nur $32 \mathrm{~kg}$, während der linke Stoßzahn $225 \mathrm{~cm}$ lang ist. Das Skelett gehört einem vollausgewachsenen Mamnutmännchen an.

Nach osteologischen Merkmalen gehört das Taimyr-Mammut 1948 der spätsibirischen Form von Elephas primigenius beresovkius ( $\mathrm{H}_{\mathrm{AY}} 1922=$ E. primigenius sibiricus Depéret \& MAYET) an, die zum ersten Male 1900 am Fluß Beresovka, Nordostsibirien, gefunden $\operatorname{war}^{2}$ ). Der Schädel des Taimyr-Mammuts ist mit den Stoßzähnen in normaler Stellung gefunden worden. Die spiral gebogenen Stoßzähne verlaufen bei Austritt aus den Alveolen zunächst nach unten und außen und steigen dann nach oben, wobei ihre Endspitzen einwärts gebogen und gegeneinander gerichtet sind. Dieselbe Lage der Stoßzähne wurde bei dem uralischen Mammut aus der Umgebung des Dorfes Purtovo, beim Beresovka-Mammut und bei einem Mammut von der Insel Ljachowski beobachtet. Das Skelett des Taimyr-Mammuts ist jetzt in der Halle für fossile Elephanten des Zoologischen Museums der Akademie der Wissenschaften ausgestellt, wo auch der Vertreter. der älteren Form des sibirischen Mammuts - Elephas primigenius giganteus Brandt - zu finden ist (Gargutt \& Dubinin). (Vgl. Abb. 2).

Aus dem Torfgestein, welches an den Kadaverteilen haftete, sind von Tichomirof: (1950) folgende Pflanzenreste bestimmt worden:

$$
\text { Gef } \ddot{a} ß \mathrm{pflanzen}
$$

Dryas punctata Juz., Carex hyperborea Drej., Eriophorum angustifolium Roth., Salix arctica Pall., S. polaris Wablb., Cassiope tetragona (L.), Luzula sp.

\section{L a u b - und L e bermoose}

Cirriphylum cirrosum (SchwaEgr.) Grout., Drepanocladus Sendtneri (Schimp.) Warnst., D. intermedius (Lindb.) Warnst., Camptothecium trichoides Broth., Tortella

$\left.{ }^{2}\right)$ Unter den physisch-geographischen Verhältnissen in Nordsibirien hat sich hier eine neue Mammutrasse entwickelt, die sich vom typischen europäischen Elephas primigenius BLum. unterscheidet (Tolmachoff, Depéret, Hay). 
tortuosa (L.) LIMPR., Distichium montanum (LAM.) HAG., Hylocomium splendens (Hedw.) BR. \& SCH., Polytrichum juniperinum Willd., $P$. alpinum Hedw., Campylium Zemliae C. Jens., Oncophorus Wablenbergi Brid., Meesea trichoides (L.) SPRuce, Encalypta rhabdocarpa Schwaegr., Paludella squarrosa (L.) BRID., Blepharostoma trichophyllum (L.) Dum., Cephaloziella divaricata (Franc.) Schiffn., Sphenolobus minutus (Crantz) StepH., Scapania sp., Plagiochila sp.

Diese Pflanzenarten sind auch in der rezenten Flora von Taimyr vertreten, wobei einige von ihnen als Edifikatoren oder Subedifikatoren der Pflanzenassoziationen im Bereich der Fundstätte auftreten, wie z. B. Dryas punctata, Eriophorum angustifolium, Cassiope tetragona, Salix arctica und S. polaris und Carex byperborea. Bei den aufgezählten Pflanzen sind neben den oberirdischen Teilen auch ziemlich deutlich ausgebildete Wurzelreste erhalten geblieben, welche bis zu gewissem Grade auf ihr Wachstum an Ort und Stelle hindeuten. Auffallend ist, dal3 eine und dieselbe Torfprobe Pflanzenarten verschiedenen ökologischen Charakters enthält. Neben ausgesprochen wasserliebenden Pflanzen finden sich auch solche, die auf trockenen Stellen wachsen. Diese rätselhafte Vermischung der Pflanzenarten verschiedener Lebensweise findet nach TicноMIROFF eine Erklärung, wenn man sich die Flora der sog. tetragonalen flach-hügeligen (Eriophorum-Riedgras-Moos-)Sümpfe genauer anschaut, die heute im Bereich der Mammutfundstätte verbreitet ist. Man findet auch in diesen Sümpfen eine ungewöhnliche Kombination verschiedener ökologischen Formen. Auf etwas gehobenen, flachen Hügeln wachsen hier mäßig wasserliebende Pflanzen; die tieferen Stellen liegen unter Wasser und sind von wasserliebenden Pflanzen eingenommen. Die Vegetation am Fluß Mamontovaja war nach Tichomiroff (1950) zur Zeit des Unterganges des Taimyr-Mammuts in ihren Grundzügen der Flora der rezenten tetragonalen, flach-hügeligen Sümpfe ähnlich. Die Torfbildung ging jedoch damals im Gebiet der Fundstätte intensiver vor sich als in der Gegenwart.

Über die Lebensweise und Ursachen des Aussterbens des sibirischen Mammuts liegen widersprechende Auffassungen vor. Von größter Bedeutung für das Leben des Mammuts als pflanzenfressenden Tieres war natürlich die Vegetation in seinem Lebensraum. Von entscheidender Bedeutung für die Beurteilung der Nahrung des Mammuts ist die Arbeit von Sukatschew. In dieser, in der ganzen Weltliteratur über das Mammut einzigartigen Arbeit hat Sukatschew mit außerordentlicher Sorgfalt die unverdauten Pflanzenreste aus dem Magen eines Mammutkadavers beschrieben, der 1900 am Fluß Beresovka, einem rechten Zufluß der Kolyma, etwa $320 \mathrm{~km}$ nordöstlich der Stadt Sredne-Kolymsk (Jakutien) gefunden worden war (nähere Einzelheiten darüber bei Pfizenmayer und Tolmachoff). Sukatschew kam bei seinen Untersuchungen zur wichtigen Erkenntnis, daß das sibirische Mammut sich nicht von Koniferen-Zweigen und -Zapfen ernährte, wie man seit der 2. Hälfte des 19. Jahrhunderts glaubte (K. E. v. BAER, J. F. BRANDT), sondern hauptsächlich von Wi es eng räs e rn. Da im Mageninhalt auch gereifte Früchte von Gräsern anwesend waren, hat SuKatschew den Schluß gezogen, daß das Beresovka-Mammut wahrscheinlich in der zweiten Hälfte des Sommers starb. Die im Norden weit verbreiteten Moose spielten in der Nahrung des Mammuts wegen ihres geringen Nährwertes wahrscheinlich nur eine untergeordnete Rolle, wie z. B. bei den rezenten Rentieren, welche die Moose nur in Zeiten des Futtermangels fressen (Tolmachoff). Auch im Magen einer Mammutleiche von der Insel Gr.-Lachowski wurden Gräser und Riedgräser festgestellt. Die Anpassung des Mammuts an die beschriebene Nahrung wurde später von ganz anderer Seite bestätigt. Die Untersuchung eines einzigen bis jetzt bekannt gewordenen Mammutrïssels (Unikum!) vom Fluß Gr.-Baranicha im Kolyma-Becken (Jakutien) zeigte nämlich, daß dieser Rüssel einen stark entwickelten unteren Lappen und einen oberen fingerartigen Fortsatz besaß. Auf diese Weise stellte das Ende des Rüssels ein ausgezeichnetes Greiforgan zum Abreißen von Gräsern und Moosen dar (FLERov). 
Die bisher gesammelten Erfahrungen lassen weiterhin eine gewisse, durch die Jahreszeiten bedingte Periodizität in der Nahrung des Mammuts feststellen. Man muß der Auffassung von Tolmachoff recht geben, daß die Nahrung des Mammuts im Winter aus Blättern, kleinen Zweigen und Baumrinde sowie aus Flechten bestand, dagegen im Sommer vorzugsweise ähnlich wie beim Rentier aus grünem Grasfutter. Ferner nimmt Tolmachoff an, daß das Mammut ein Nomadenleben führte. Im Frühling wanderte es aus der Waldzone in die südlichen oder sogar nördlichen Teile der Tundra und im Herbst in umgekehrter Richtung, ähnlich den Rentieren in der Gegenwart. Diese dringen im Frühjahr und Anfang Sommer aus Gebirgsbezirken und südlichen Teilen der Waldtundra bis an die Polarküste Sibiriens vor, wobei sie bis $1000 \mathrm{~km}$ in einer Richtung zurücklegen. Die Größe des Mammutkörpers verlangt eine entsprechend ausreichende Nahrung (der Magen des Beresovka-Mammut enthielt $12 \mathrm{~kg}$ Nahrung), was einen dauernden Aufenthalt des Mammuts in hocharktischen Regionen in der Umgebung von Gletscherdecken mit kärglicher Vegetation ausschließt. In diesen Regionen hielt es sich wahrscheinlich nur im Sommer auf, wo es Schutz vor blutsaugenden Insekten suchte (TICHOMiroff 1951). Den ungewöhnlichen Reichtum der Insel Gr.-Ljachowski im Nordsibirischen Archipel an Elfenbein kann man ebenfalls durch diese Migrationen des Mammuts erklären. In der Mammutepoche erstreckte sich das sibirische Festland weiter nördlich als heutzutage, und die genannte Insel stellte einen Teil des Festlandes dar. Die Mammutherden konnten daher im Sommer bis zu den Weiden der heutigen Insel Gr.-Ljachowski wandern (OBRUTschew).

Das stratigraphische Profil der Ablagerung der ersten Terrasse des Flusses Mamontovaja, in die das Mammut von Taimyr ursprünglich eingebettet war, ist paläontologisch noch ungenügend untersucht. Jedoch weisen schon die bis jetzt gesammelten Beobachtungen darauf hin, daß auf der Taimyr-Halbinsel zu Lebzeiten des Mammuts eine stärker wärmeliebende Flora existierte als in der Gegenwart. In schlammigen Ablagerungen der Terrasse wurden zahlreiche Reste (Blätter, Hölzer) der Zwergbirke (Betula exilis Suk.), ebenso Reste von Weiden (Salix arbıscula L. oder S. lanata L.) von großen Ausmaßen (5-6 cm im Durchmesser, 2-2,5 m lang) sowie Wurzelstöcke von Equisetum arvense gefunden. Die Zwergbirke fehlt jetzt in der Umgebung der Fundstätte gänzlich. Die großstrauchigen Weiden finden sich gegenwärtig nur im Gebiet der Chatanga-Waldtundra, also viel weiter südlich der Fundstätte. Dieses Vordringen von Holzpflanzen in die nördlichen Breiten auf Taimyr in der Vergangenheit wird auch durch den Fund von Larix-Resten in den nacheiszeitlichen Ablagerungen bis zur Breite von $74^{\circ} 40^{\prime}$, d. h. fast um $2^{\circ}$ nördlicher als in der Jetztzeit, bestätigt. Andererseits sind in der russischen Literatur viele Hinweise vorhanden, die zum Schluß drängen, das das Aussterben der letzten sibirischen Mammute in der Nacheiszeit stattgefunden hat. Auf Grund dieser Tatsachen spricht TICHомiroff (1951) die Vermutung aus, daß in einem Zeitabschnitt des Postglazials im Gebiet, wo sich die Reste des sibirischen Mammuts finden, physisch-geographische Bedingungen herrschten, die den heutigen Verhältnissen nahe dem Nordrande der Waldtundra (Übergangszone zwischen der Wald- und Tundra-Zone) oder des Südrandes der Tundra ähnlich waren. Die Auen von Flüssen waren wahrscheinlich von großstrauchigen Weiden vom Typus Salix arbuscula und $S$. lanata und grasartigen Gewächsen bedeckt. Die ersten Aueterrassen waren infolge Auftauens des Dauerfrostbodens von Sümpfen eingenommen, in denen die Torfbildung rascher vor sich ging, als es gegenwärtig in diesen Breiten der Fall ist. Auf Hügeln wuchsen Zwergbirken und auf südlichen Hängen vielleicht auch Lärchen. Die vorherrschenden Pflanzenarten waren jedoch verschiedene Gräser und Riedgräser.

In der Zusammensetzung der Flora seit dem Untergang des Taimyr-Mammuts und bis zur Gegenwart haben somit wesentliche Veränderungen stattgefunden. Diese bestehen im allmählichen Verschwinden der Wald- und Straucharten und in der Zunahme 
der Tundra-Pflanzenassoziationen des rezenten Typus. Daraus zieht Tichominoff (1951) den Schluß, daß das Taimyr-Mammut in einem wä rmeren Klima lebte als dem Klima der Gegenwart. Man kann nach TIсноміRoff die Existenz des Taimyr-Mammuts zeitlich mit der Waldphase in der nacheiszeitlichen Geschichte der nordsibirischen Vegetation parallelisieren, welche einer bedeutenden Erwärmung des Klimas (dem postglazialen Klimaoptimum) entsprach. $\mathrm{Zu}$ dieser Zeit drangen die Wälder bzw. einzelne Baumgruppen um $2.5^{\circ}$ bis $3.5^{\circ}$ nordwärts von der heutigen Grenze vor. Während dieses wärmeren Zeitabschnittes lebten die letzten Vertreter der sibirischen Mammute als die charakteristischsten Repräsentanten der Postglazialzeit. Man darf aber das sibirische Mammut in keinem Falle als wärmeliebendes Tier betrachten. Im Gegenteil sprechen die lange Haarkleidung (bis $\mathrm{zu} 50 \mathrm{~cm}$ an Brust und Schultern), eine dicke Schicht von über den ganzen Körper gleichmäßig verteiltem Fett und andere Merkmale für die Anpassung des Mammuts an die harten Lebensbedingungen in den nördlichen Teilen Sibiriens. Die stark entwickelten und spiral gebogenen Stoßzähne dienten, wie neuerdings von GARgutT und DuBININ begründet wurde, als Werkszeug zum Wegräumen von Schnee.

Die neuerdings von $Z_{\text {AKLINSKAJA }}$ durchgeführten sporen- und pollenanalytischen Untersuchungen der Sedimente der 1. und 2. Terrasse lassen erkennen, daß zur Zeit ihrer Entstehung auf Taimyr eine baumlose Vegetation mit auffallend vorherrschenden Wiesengräsern existierte. Diese Tatsache darf übrigens als eine weitere Bestätigung der Auffassung von Sukatschew betrachtet werden, nach der die Wiesengräser die Hauptnahrung des sibirischen Mammuts darstellten. Ferner drängen diese Untersuchungen zur Annahme, daß das Taimyr-Mammut ursprünglich anscheinend in den Ablagerungen der 2. Aueterrasse begraben war, ehe seine Leiche auf das Ufer des Flusses Mamontovaja herabstürzte. Hinsichtlich des Alters des Taimyr-Mammuts kam Z Z Erkenntnis, daß es lebte und starb nicht während des postglazialen Klimaoptimums, wie Tichomiroff glaubt, sondern kurz vor dem Eintreten dieses Optimums.

Die Annahme des postglazialen Alters für das Taimyr-Mammut steht an sich nicht im Widerspruch zu den in Sibirien gesammelten Erfahrungen. Die Vegetation zur Zeit des Beresovka-Mammuts war nach Sukatschew der rezenten Flora von Nord-Jakutien ähnlich. Neuerdings unterschied P. A. NikTiN (1939) drei verschiedenalterige Mammut-Floren, nämlich eine Präwürm-, eine Würm- und eine Postwürm-Flora ${ }^{3}$ ). Nach STRACHOw hat es in Nordasien mindestens zwei Vereisungen gegeben, von denen die ältere, wahrscheinlich mit der Riß-Eiszeit synchron, am stärksten war. In der Interglazialzeit hat hier eine boreale marine Transgression stattgefunden, die augenscheinlich analog und synchron mit derjenigen im Norden der Russischen Tafel war. Hier lebte das Mammut noch in der Zeit des Yoldia-Meeres und starb in der Ancylus-Zeit aus. In Nordasien existierte das Mammut nach STRAchow in der Würmzeit und s päter, anscheinend bis in die geologisch jüngere Zeit hinein. Meinerseits möchte ich die Vermutung aussprechen, daß das Mammut vielleicht weit gïnstigere Lebensbedingungen in Nordasien fand als anderswo in der Welt, wofür zahlreiche Funde seiner Reste sprechen, so daß seine letzten Vertreter in Sibirien erst im Laufe der Postglazialzeit ausstarben.

Nun liegt eine neuere Bestimmung des Alters des Taimyr-Mammuts vor, die nicht unerwähnt bleiben darf ${ }^{4}$ ). In seinem interessanten Artikel über die Verwendung man* cher Isotopen für die Lösung verschiedener geologischer Probleme erwähnt WINORgADow kurz, daß A. W. Trofimow in seinem Institut mit Hilfe des Isotops $\mathrm{C}^{14}$ das absolute

3) Die einschlägigen Literaturangaben hierzu s. bei Tiсномівоw (1950).

$\left.{ }^{4}\right)$ Für den Hinweis auf die Untersuchungen von A. Trofimow bin ich Herrn Prof. Dr. H. Gams, Innsbruck und Herrn Dr. H. GRoss, Bamberg, zu Dank verpflichtet. 
Alter des Taimyr-Mammuts mit ca. 12000 Jahren (= Beginn der Alleröd-Wärmeschwankung in Europa) bestimmt. Eine Stellungnahme von Geologen bzw. Paläobotanikern zu dieser Datierung liegt noch nicht vor.

\section{Schriftenverzeichnis}

Sukatschew, V. N.: Untersuchung der Pflanzenreste aus der Nahrung einer Mammutleiche, gefunden am Fluß Beresovka in Jakutien. - Wiss. Erg. einer Expedition der Akad. d. Wissenschaften, Bd. 3, Petrograd 1914.

HAY, O. P.: Observations on some extinct Elephants. - Washington, D. C. 1922 (im Original mir nicht zugänglich).

Depéret, Ch., Mayet, L.: Monographie des eléphants pliocènes de l'Europe et de l'Afrique du Nord. - Ann. Univ. Lyon, N. S. 43, S. 89-224, 1923.

Pfizenmayer, E. W.: Mammutleichen und Urwaldmenschen in Nord-Ost-Sibirien. - 341 S., Leipzig 1926.

Tolmachoff, I. P.: The carcasses of the mammoth and rhinoceros in the frozen ground of Siberia. - Trans. Amer. Phil. Soc. N. S. 23, S. 1-74. 1929.

Flerov, C. C.: Trunk of mammoth found in the Kolyma District, Siberia. - C. r. URSS, Serie 7, 6, 1931.

TiсномirofF, B. A.: Zur Charakteristik der Pflanzendecke während der Mammut-Epoche auf Taimyr.-Botanitscheskij Zhurnal 35, S. 482/97. 1950.

Tichomiroff, B. A.: Über die Vegetation zur Mammut-Zeit in Nord-Sibirien. - Priroda 40, S. 33-40. 1951.

Gargutt, B. E., Dubinin, V. B.: Ưber das Skelett des Taimyr-Mammuts. - Zoologitscheskij Zhurnal 30, S. 17-22. 1951.

Portenko, L. A., Tichomiröff, B. A., Popov, A. I.: Die ersten Resultate der Ausgrabung des Taimyr-Mammuts und die Erforschung seiner Einbettungsbedingungen. - Zoologitscheskij Zhurnal 30, S. 3-22. 1951.

Obrutschew, V. A.: Notiz in: Priroda 41, S. 120/22. 1952.

Pavlovskij, E. N.: Taimyr-Expedition zur Ausgrabung der Mammutleiche. - Vestnik der Akad. d. Wissenschaften 20, S. 126/28. 1950 .

ZakLinskaja, E. D.: Zur Frage der Pflanzendecke zur Zeit des Taimyr-Mammuts. - Doklady Akad. Nauk SSSR 98, S. 471-74. 1950.

Strachow, N. M.: Grundlagen der historischen Geologie [russ.], Bd. 2, Moskau-Leningrad 1948, S. 306-309.

Winogradow, A. P.: Geochemie der Isotopen. - Izv. Akad. Nauk SSSR ser. geol. 1954, Nr. 3, S. 3-9; Vestnik Akad. Nauk SSSR, 1954, Nr. 5, S. 26-43.

Manuskr. eingeg. 13. 9. 1954.

Anschrift d. Verf.: Dr. N. Polutoff, Gmelin-Institut Tübingen, Wilhelmstraße 26. 